$3{ }^{1}$ Targeted Therapeutics, Department of Biomaterials Science and Technology, Faculty of 4 Science and Technology, University of Twente, Enschede, The Netherlands

$5 \quad{ }^{2}$ ScarTec Therapeutics BV. Enschede, The Netherlands

6 *corresponding author: j.prakash@utwente.nl

7 KEYWORDS: Pancreatic ductal adenocarcinoma, pancreatic cancer, tumor stroma, cancer8 associated fibroblasts, desmoplasia 
Pancreatic stellate cells (PSCs) are the major contributor to the aggressive, metastatic and resilient nature of pancreatic ductal adenocarcinoma (PDAC), which has the worst prognosis with 5-year survival rate of $8 \%$. PSCs constitute more than $50 \%$ of the tumor stroma in PDAC, where they induce extensive desmoplasia by secreting abundant extracellular matrix proteins. In addition, they also establish dynamic crosstalk with cancer cells and other stromal cells, which collectively support tumor progression via various inter-and intra-cellular pathways. These cellular interactions and associated pathways may reveal novel therapeutic opportunities against this unmet clinical problem. In this review, we will discuss the role of PSCs in inducing tumor progression, their crosstalk with other cells, and therapeutic strategies to target PSCs.

\section{Pancreatic ductal adenocarcinoma}

Pancreatic ductal adenocarcinoma (PDAC) is the most common form of pancreatic cancer that represents more than $90 \%$ of all pancreatic cancer types $[1,2]$. Though the number of incidences for PDAC is rather low accounting for only $2 \%$ of all cancers, the mortality rate is tremendously high causing the 5-year survival rate of $8 \%$, attributed to the rapid development of advanced disease or metastasis $[3,4]$. The standard-of-care therapy for PDAC is combination chemotherapy, FOLFIRINOX, or gemcitabine plus nab-paclitaxel. These therapies, however, fail to show much benefits to PDAC patients. The aggressiveness of PDAC and the limited response to chemotherapies are attributed to the highly desmoplastic microenvironment. The tumor microenvironment (TME) in PDAC, which is often known as tumor stroma, can occupy up to $90 \%$ of the entire tumor mass [5]. Pancreatic stellate cells (PSCs) are the most prominent cell type with in the PDAC stroma, constituting about $50 \%$ of it. As a key player within the TME, pancreatic stellate cells (PSCs) have received enormous attention in the field of therapeutics against PDAC. The dynamic crosstalk between PSCs and cancer cells as well as PSCs' role in generating desmoplasia have already been well established [6-8]. Emerging literature has now unravelled new biological processes related to PSC induced tumor progression, survival and therapeutic escape mechanisms in PDAC [9-12]. These new insights will, in the near future, fuel the development of therapeutics against PDAC and support for the better clinical outcomes. In this review, we comprehensively discuss the 
biological standing of PSCs in PDAC, their interaction with other cell types, molecular mechanisms controlling their phenotype and therapeutic strategies to target them.

\section{Pancreatic stellate cells (PSCS) in PDAC}

PSCs are star-shaped stromal cells located at the basolateral aspect of acinar cells or surrounding peri-vascular and peri-ductal regions in the healthy pancreas [6]. Quiescent PSCs are involved in the storage of vitamin A rich lipid droplets, normal exocrine and endocrine secretion, phagocytosis, immunity and maintenance of normal stroma composition [13]. During the development of PDAC, quiescent PSCs get activated via various underlying mechanisms due to the influence of risk factors (ethanol and its metabolites, chronic inflammation and smoking), environmental stress (e.g. hypo-perfusion, hypoxia, oxidative stress), cellular secretory factors (e.g. interleukin-1 (IL-1), interleukin-6 (IL-6), hypoxiainducible factor 1-alpha (HIF1 $\alpha$ ), transforming growth factor-beta (TGF- $\beta$ ), connective tissue growth factor (CTGF)), and molecular signaling pathways (e.g. Wnt/ $\beta$-catenin signaling, PI3K pathway) [14]. The activated PSCs (aPSCs) lose cytoplasmic vitamin A storing lipid droplets and express $\alpha$-smooth muscle actin ( $\alpha$-SMA) and large amounts of extracellular matrix (ECM) [14, 15]. $\alpha$-SMA expression on aPSCs has been directly correlated with PDAC clinic-pathological characteristics and is known as an independent positive prognostic parameter [14, 15]. aPSCs possess a proliferative, migratory phenotype and induce desmoplasia by synthesizing abundant ECM components such as collagens, fibronectin, laminin and hyaluronic acid and unbalanced expression of matrix-metalloproteases (MMPs) and tissue inhibitors of metalloproteinases (TIMPs) (Figure 1) [6, 14]. Additionally, aPSCs secrete increased levels of cytokines such as interleukin-1, $-6,-8$ and -10 (IL-1, $-6,-8$ and -10$)$ and growth factors, including insulin-like growth factor 1 (IGF1), vascular endothelial growth factor (VEGF), and platelet derived growth factor (PDGF), fibroblast growth factor (FGF), connective tissue growth factor (CTGF) and C-X-C motif chemokine 12 (CXCL12) [6, 7]. These cytokines and growth factors promote angiogenesis, and proliferation, migration and invasion of epithelial cancer cells that leads to metastasis $[6,8,14]$.Furthermore, PSCs-secreted soluble factors, especially IL-6, has been shown to be involved in transitioning of non-invasive into invasive PDAC, and to drive immunosuppression in the TME by promoting the accumulation of myeloid-derived suppressor cells (MDSCs), via STAT3-dependent mechanism [16, 17]. Moreover, cancer cells also secrete cytokines such as IL-1, IL-6 and TNF- $\alpha$, and growth factors including TGF- $\beta 1$, PDGF- 
BB [2]. These reciprocal interactions between cancer cells and aPSCs contribute to the progression of PDAC significantly. Interestingly, Sousa et. al., have shown that PDAC cells induce autophagy in PSCs to secrete alanine to sustain PDAC cells metabolic needs and growth in the nutrient-deprived pancreatic cancer environment [18]. More recently, Hessmann et. al. have demonstrated that PSCs actively contribute to drug resistance of pancreatic tumors by entrapping gemcitabine within their cytoplasm and thereby limiting the effect of gemcitabine on pancreatic cancer cells [11]. These studies suggest the reciprocal communication between tumor cells and PSCs support PDAC growth and aggressiveness.

a

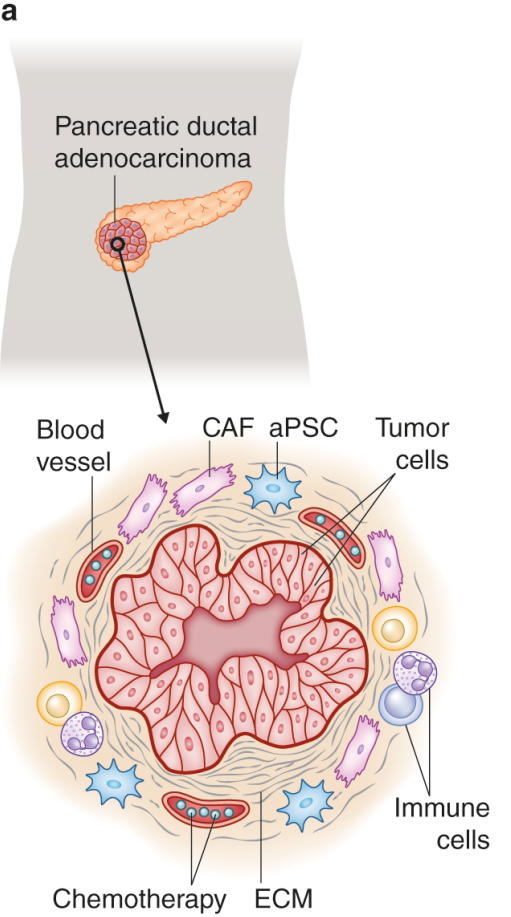

b

TGF- $\beta$, PDGF, periostin,

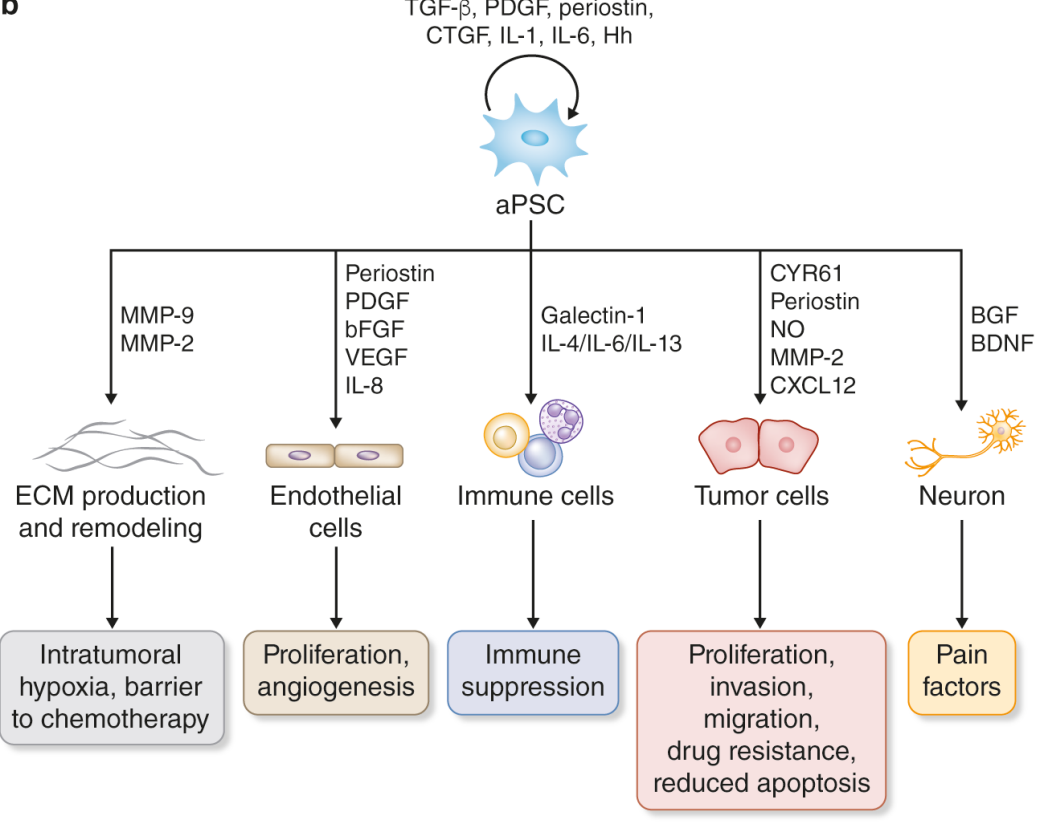

Figure 1. Role of activated pancreatic stellate cells (aPSCs) in pancreatic ductal adenocarcinoma (PDAC). (a) The zoomed image of tumor shows the arrangement of ductal tumor cells in PDAC (inside) surrounded by tumor stroma containing cancer-associated fibroblasts CAFs (or aPSC) and extracellular matrix (ECM) as well as blood vessels. CAFs are mainly derived from PSCs. Tumor stroma acts as a barrier to chemotherapy which cannot penetrate through the thick stroma layers. (b) aPSCs act in an autocrine and a paracrine manner by secreting several growth factors and cytokines which activate themselves and other stromal cells. aPSCs also produce abundant extracellular matrix (ECM) proteins and remodel it.

Abbreviations: TGF- $\beta$, transforming growth factor-beta; PDGF, platelet-derived growth factor; CTGF, Connective tissue growth factor; IL-1,-4,-6,-8,-13 Interleukin-1,-4,-6,-8,-13; Hh, hedgehog; bFGF, basal fibroblast growth factor; VEGF, vascular endothelial growth factor; CYR61, cysteine-rich angiogenic 
inducer 61; NO, nitric oxide; MMP-2, matrix metalloproteinase-2; CXCL-12, C-X-C Motif Chemokine Ligand 12; BGF, bone growth factor; BNGF, beta-nerve growth factor.

\section{Differences in PSC activation in PDAC versus pancreatic fibrosis}

PSCs differentiate into myofibroblasts in pancreatic fibrosis and PDAC $[19,20]$. PSCs have been shown to play a crucial role in pancreatitis [21] leading to fibrosis. However, the major question that remain unanswered is whether PSCs differentiate differently and contribute to pancreatic fibrosis and PDAC. During pancreatitis, PSCs are mainly recruited, to a larger extent, from resident PSCs and to a lesser extent from bone marrow [2]. aPSCs were found to be present at the early stages of acute pancreatitis and contribute to gland repair and recovery without residual pathological fibrosis [22].The absence of fibrosis in acute pancreatitis was attributed to the bile acid-induced necrosis of aPSCs [23]. However, chronic pancreatitis presents with a massive amount of fibrosis related to aPSCs, as shown by the overexpression of nerve growth factor (NGF), selective marker for aPSC [24]. During chronic pancreatitis, PSCs are activated [14] by acinar cells in a paracrine manner through the secretion of TGF- $\beta$ [25]. Additionally, cytokines, reactive oxygen species (ROS) and oxidative stress in the fibrotic areas of pancreatitis contributes to PSC activation [14]. Prominently, it has been demonstrated that progression of chronic pancreatitis is closely associated with crosstalk between alternatively activated macrophages (AAMs) and PSCs, whereby PSCs have been suggested to be a source of IL-4/IL-13 resulting in the activation of AAMs and fibrosis progression [26]. The proliferation of aPSCs in chronic pancreatitis is likely due to increased expression of the mitogen plateletderived growth factor receptor (PDGFR) [27]. Furthermore, chronic pancreatitis poses a high risk for PDAC development, indicating a role of the fibrotic microenvironment in PDAC progression [4]. Binkley et al. have found 107 genes that are commonly expressed in the stromal cells of patients with PDAC or with chronic pancreatitis [28]. Additionally, in PDAC, aPSCs were found in pre-invasive ductal lesions surrounding stroma, in pancreatic intraepithelial neoplastic lesions (PanIN) and invasive carcinomas with chronic pancreatitis [29].

In PDAC, aPSCs are confronted with additional growth factors secreted by malignant cells and other stromal cells which are already educated by malignant cells. This can lead to generation of several differently activated PSCs. However, research defining PSC activation stages and their differentiation into different cancer-associated fibroblasts (CAFs) populations is just 
121 evolving. A recent study by Ohlund et al. made a first step in defining functionally different 122 CAF subtypes, originated from aPSCs, named myofibroblastic CAFs (myCAFs) and 123 inflammatory CAFs (iCAFs) [30]. MyCAFs show elevated levels of $\alpha$-SMA expression and are 124 located in close proximity to neoplastic cells, while iCAFs are located more distant from 125 neoplastic cells, lack $\alpha$-SMA expression but secrete high amounts of IL- 6 and other chemokines 126 known to support cancer progression. However, CAF populations do not seem to be limited to myCAFs and iCAFs. The authors have also revealed an additional population of CAFs which are negative for both, $\alpha$-SMA and IL6, indicating heterogeneity of CAFs. Very recently, the group of Tuveson has demonstrated that IL-1 is responsible for generating iCAFs by activating JAK/STAT pathway, and this process can be antagonized by TGF- $\beta$ by downregulating IL-1R1 expression which promotes differentiation into myofibroblasts [12]. Another study demonstrated the presence of two distinct populations of activated PSCs i.e. CD10 positive and CD10 negative in resected pancreatic cancer tissue. The authors showed that CD10 expression on PSCs was markedly higher in tumor tissue and was associated with positive nodal metastases and poor prognosis [31]. Franco-Barraza et al. have identified a CAF phenotype with high expression of plasma membrane-localized, active $\alpha 5 \beta 1$ integrin. They have correlated the desmoplastic traits and prognosis of neoplastic recurrence with integrin $\alpha 5 \beta 1$ expression, which has shown to be matrix-regulated by integrin $\alpha v \beta 5$ [32]. In this study, the author's proposed a novel prognostic tool, in which they used stromal localization and levels of active Smad $2 / 3$ and integrin $\alpha 5 \beta 1$ to distinguish patient-protective from patientdetrimental desmoplasia, to predict pancreatic cancer recurrence [32]. We have recently shown integrin $\alpha 5$ (ITGA5) as a prognostic marker, as its overexpression in PDAC stroma was associated with poor survival of patients [33]. Furthermore, knockdown of ITGA5 in PSCS inhibited their adhesion, migration, and proliferation and also inhibited TGF $\beta$-mediated differentiation into CAFs and PSC-induced tumor cell proliferation and migration [33].

146 These evidences underline that in pancreatic fibrosis PSCs mainly differentiate into myofibroblasts whereas in the complex microenvironment of PDAC they differentiate into different fibroblasts (CAFs) which may eventually perform a variety of functions.

\section{Role of aPSCs in PDAC pathophysiology}

PDAC develops from histologically different precursor lesions known as pancreatic intraepithelial neoplasia (PanIN), intraductal papillary mucinous neoplasm (IPMN) and 
mucinous cystic neoplasm (MCN), with decreasing frequency of development, respectively [34]. The majority of invasive PDAC develops from PanIN lesions which are characterized into PanIN-1, PanIN-2 and PanIN-3 by their degree of dysplasia [35]. Next to PanIN, IPMN lesions are precursors of invasive PDAC and therefore early detection of PanIN and IPMN lesions presents the opportunity to cure pancreatic cancer before the development of an invasive carcinoma [36]. Genetic analysis of PanIN lesions has shown increasing incidence of KRAS, p16/CDKN2A and BRAF mutations [34].

Staining of $\alpha-S M A$ indicates the presence of aPSCs surrounding PanIN lesions [37]. IL-6 secreted by aPSCs was found to activate STAT3 signaling in non-invasive, precursor PanIN cells, thereby causing enhanced cell invasion and colony formation [17]. Both, IL-6 neutralization and STAT3 inhibition resulted in attenuation of aPSC-conditioned medium induced STAT3 signaling and tumorigenicity, indicating a novel role for aPSCs in the transition of non-invasive pancreatic precursor cells into invasive PDAC [17]. In KRAS ${ }^{G 12 D}$ mice (mice with activated KRAS), high-fat and high-calorie diet and exposure to smoking compounds promoted the formation of advanced PanIN lesions with aPSCs [6].

Although aPSCs have been linked to genomic instability and are capable of inducing EMT in PDAC, PSCs effects have not been directly linked to the genetic mutation that are acquired during PDAC onset and progression, which still needs to be further investigated.

\section{Role of aPSCs in PDAC aggressiveness}

The aggressive character of PDAC possibly reflects several factors such as the highly proliferative nature of tumor cells, chemoresistance leading to inhibition of apoptosis in tumor cells, early attainment of metastatic phenotype by tumor cells, suppressed tumor immunity and poor penetration of chemotherapy to tumor cells, due to a stromal physical barrier [38, 39].

Activated PSCs contribute to almost all these factors and for simplicity their contribution can be divided into two sections i) physical interaction ii) crosstalk within stroma. The mechanisms of growth factor and cytokine crosstalk between aPSCs, tumor cells and cells of the stroma are described in the following section and are depicted in Figure 1 [38]. 
182

There are several ways by which aPSCs contribute to the aggressiveness of PDAC by limiting the efficacy of standard treatments. aPSC-induced desmoplastic reaction plays a significant role in the chemoresistance. The extensive desmoplastic reaction with an abundant amount of aPSC-secreted ECM proteins leads to intra-tumoral hypoxia and a self-perpetuating fibrosis cycle [38]. The tumoral hypoxia causes genomic instability of cancer cells leading to epithelial to mesenchymal transition (EMT), an increased malignant behavior and resistance to chemotherapy [38]. Additionally, aPSCs have been recognized to be present in metastatic nodules, which indicates their ability to intravasate and extravasate in and out of blood vessels, survive in the blood circulation and seed in the distant organs, thereby creating a metastatic niche [40]. Very recently, autophagy in aPSCs induced by environmental stress and tumor cell-stroma interactions, was reported to be associated with histological grading, peritoneal dissemination, perivascular invasion and lymph node metastasis [41].

aPSCs produce excessive amounts of ECM molecules, such as collagens, fibronectin, laminin and tenascin-C, which not only interact with PSCs but also control the tumor cell phenotype [42]. Berchtold et al. have shown an overexpression of collagen $V$ in PDAC samples, which is produced by PSCs, act as an important mediator for viability, adhesion, migration, and metastatic potential of pancreatic cancer cells regulated via $\beta 1$-integrin/FAK signaling pathway [42]. Furthermore, the densely deposited ECM acts as a physical barrier and therefore prevents drug penetration through constricted blood vessel, thereby impairing drug delivery to cancer cells [43]. Recently, another novel molecular mechanism has been proposed wherein TGF- $\beta$-activated PSCs express cysteine-rich angiogenic inducer 61 (CYR61), a matricellular protein regulating the nucleoside transporters hENT1 and hCNT3 responsible for the cellular uptake of gemcitabine [44]. This causes deprivation of gemcitabine from tumor cells leading to the treatment failure.

\subsection{Role of aPSCs in PDAC metabolic reprogramming}

aPSCs-secreted ECM contributes to dense fibrotic stroma and increased interstitial pressure [45]. As a consequence, enhanced stromal pressure results in vascular collapse, hypoperfusion, and lack of nutrient and oxygen delivery to the tumor tissue [46, 47]. Enhanced glucose metabolism via Warburg or reverse Warburg effect in cancer cells $[48,49]$, remain insufficient to compensate for tumor growth and survival. Metabolic rewiring between cancer 
cells and stromal components support the nutritional needs for tumor growth. Several studies have suggested the critical role of aPSCs in PDAC metabolic reprogramming thereby promoting PDAC progression and invasiveness under nutrient-deprived conditions [50, 51]. It has been increasingly recognized that mutual metabolic cross-talk between PDAC cells and aPSCs is a result of genetic mutations and paracrine signaling [47, 51]. Oncogenic KRAS mutation in PDAC cells has been shown to enhance glucose uptake, activate aerobic glycolysis and glutamine metabolism (source of carbon and nitrogen) via regulation of different pathways [51]. KRAS mutation induces sonic hedgehog secretion from PDAC cells to activate PSCs which in turn activate downstream PI3K-AKT pathway and increased mitochondrial respiratory activity and oxygen availability for PDAC cells under hypoxic conditions [52]. Furthermore, KRAS-mutant PDAC cells upregulate micropinocytosis to import extracellular proteins for lysosomal-mediated catabolism for fueling TCA cycle, essential amino acid recycling thereby supporting tumor growth [53]. Furthermore, PSCs-derived exosomes containing mRNA, miRNA, intracellular metabolites (amino acids, acetate, stearate, palmitate and lactate) to fuel tricarboxylic acid (TCA) cycle in PDAC cells and enhance tumor growth [54]. Strikingly, PDAC cells has been shown to increase autophagy in PSCs, mediating secretion of alanine as an alternative carbon source to glucose and glutamine, thereby compensating PDAC cells nutritional needs via Ser/Gly, lipid and NEAAs biosynthesis [18]. Altogether, aPSCs, via metabolic cross-talk with PDAC cells, play a significant role in PDAC progression under nutrient-deprived environment.

\subsection{PSC crosstalk within stroma}

\subsubsection{Crosstalk with tumor cells}

Within the tumor stroma, growth factors, chemokines, cytokines, miRNAs and exosomes secreted by PSCS are known for their ability to act in an autocrine fashion, resulting in PSC activation or exert paracrine signals on epithelial tumor cells to increase the proliferation, migration, and invasion of tumor cells [10, 55-57]. Additionally, paracrine factors secreted by aPSCs protect tumor cells from apoptosis, radiotherapy, and chemotherapy [58]. Activated PSCs are capable of secreting nitric oxide which in turn enhance IL-1 $\beta$ expression in PDAC cancer cells in a paracrine fashion [59]. The autocrine IL-1 $\beta$-dependent pathway in cancer cells is related to the chemoresistance. Secretion of aPSC-specific periostin sustains the activity of 
aPSCs and increases PDAC cancer cells resistance to chemoradiation [49]. Periostin is also associated with a poor prognosis of PDAC and promotes PDAC cancer cell proliferation and metastasis via the epidermal growth factor receptor (EGFR)-Akt and EGFR-extracellular signal regulated kinase-c-Myc pathways [49]. Moreover, periostin silencing was found to be associated with an inhibition in gemcitabine resistance in vitro and in vivo [60]. The radioresistance effect of aPSCs is mostly dependent on integrin- $\beta 1$-FAK signaling, since abrogating this pathway decreases aPSC-mediated protection of PDAC cancer cells against radiation [61].

The increased ECM deposition in pancreatic cancers results from the paracrine stimulation of PSCs by cancer cells [14]. Furthermore, we and others have shown that co-injection of PSCS and tumor cells (e.g. PANC-1, BxPC3, MiaPaCa2) in an orthotopic models exhibited increased tumor growth as compared to subcutaneous tumors consisting solely of tumor cells, suggesting crucial role of PSCs in supporting and promoting pancreatic cancer [33, 62-65]. We have shown that subcutaneous tumors formed with PANC-1 and PSCs with ITGA5 knockdown develop smaller and less fibrotic tumors when compared to tumors formed with PANC-1 and normal PSCs in mice [33]. In PDAC, PSCs have shown to possess ECM remodeling capabilities via matrix contraction and increasing alignment and thickness of collagen fibrils [66-68]. Several studies have demonstrated the importance of ECM remodeling and stiffness in pancreatic tumor growth, PSCs activation, PSCs and PDAC cells migration and invasion $[66,68$ 70]. Next to inducing the desmoplastic reaction in PDAC, aPSCs promote metastasis and invasion in PDAC, by the induction of EMT in epithelial tumor cells [71]. Recently, we shown that integrin $\alpha 11$, a collagen binding receptor, is overexpressed in PDAC stroma and plays a key role in controlling PSC activation by TGF- $\beta$ or tumor cells and also PSC-mediated tumor cell migration and invasion [72]. In co-cultures of PSCs and tumor cells, tumor cells attain EMT characteristics such as reduced cell-to-cell contacts, a scattered and fibroblast-like shape, increased migration as well as loss of epithelial markers (e.g. e-cadherin, cytokeratin-19 and membrane-associated $\beta$-catenin) and gain of mesenchymal markers (e.g. Snail and vimentin) [73]. Indications for aPSC-induced EMT in cancer cells in vivo has been demonstrated by a decreased expression of e-cadherin and increased expression of vimentin and $\mathrm{n}$-cadherin at the invasive front of PDAC, where cancer cells get exposed to the signals from stromal cells [71]. Furthermore, EMT has also been related to chemoresistance, another factor by which PSCs contribute to the PDAC drug resistance [14]. More recently, galectin-1-induced 
275 upregulation of stromal derived factor (SDF-1), also known as C-X-C motif chemokine 12 276 (CXCL12) in aPSCs was shown to promote pancreatic cancer metastasis [9]. Next to the ability 277 of PSCs to increase the metastatic potential of PDAC cancer cells, PDAC cells secrete PDGF, which is a chemotactic factor that potentially regulates the role of PSCs in the metastatic 279 niche.

Several studies have identified that cancer stem cells, within the pancreatic tumor possess highly tumorigenic, chemo-resistant and metastatic phenotypes leading to post-operative recurrence, re-growth of therapy-resistant tumors and metastasis respectively [74-79]. Hamada et al., have demonstrated that PSCs enhances cancer stem cell-like phenotypes in pancreatic cancer cells based on increased expression of stem-cell related genes such as ABCG2, nestin, and LIN28 suggesting a role of PSCs in development of the cancer stem cell niche [80].

\subsubsection{Crosstalk with immune cells}

In PDAC, aPSCs, cancer-infiltrating macrophages, immunosuppressive myeloid-derived suppressor cells (MDSCs), mast cells and regulatory T-cells, secrete increased levels of immunosuppressive cytokines, such as IL-10 and TGF- $\beta 1$ which inhibit the activation of dendritic cells thereby suppressing immune responses and inducing immune tolerance [81]. MDSCs are highly elevated in the peripheral blood samples and in pancreatic tumor microenvironment and are associated with a poor prognosis in PDAC patients $[82,83]$. PSCs potentially drive expansion and differentiation of MDSC which promotes an immunosuppressive microenvironment via IL-6/STAT3 pathway driving immune escape and resistance to immunotherapy $[16,84]$. In pancreatic cancer, obesity is associated with increased desmoplasia [85]. In this context activation of PSCs has been induced by tumorassociated neutrophils (TAN) which are recruited by IL-1 $\beta$, secreted by adipocytes [85]. Additionally, macrophages were shown to activate PSCs via hypoxia inducible factor 1 (HIF-1) secretion [57]. aPSCs are also known to modulate the proliferation and apoptosis of effector T-cells, block T-cell activation, induce T-cell death, retaining T-cells within an anergic state within the tumor and skew the cytokine secretion towards a T helper type 2 (Th2) immune response via the secretion of Galectin-1 [86]. Ene-Obong et al. showed that activated PSCs reduced migration of $\mathrm{CD} 8(+) \mathrm{T}$ cells to juxtatumoral stromal compartments and thereby prevented their access to cancer cells [87]. Instead of that, activated PSCs attracted these T 
cells towards themselves by secreting CXCL12 and this process prevented an effective antitumor immune response [87]. Xue et al. have demonstrated that aPSCs secrete IL-4 and IL-13 which transform macrophages into alternatively activated M2 macrophages, which in turn activate PSCs by secreting TGF- $\beta$ and PDGF [26]. Furthermore, they demonstrated that intervening into IL-4/IL-13 pathways could turn-off this feed forward process, which could be an interesting pathway for developing therapeutics.

\subsubsection{Crosstalk with endothelial cells}

Activated PSCs produce a number of pro-angiogenic factors, including VEGF, bFGF, IL-8, PDGF and periostin, but also MMP-9 which contributed to blood vessel formation by decomposing the basement membrane [71]. VEGF promotes endothelial cell proliferation, survival and permeability, thereby inducing angiogenesis [71]. Periostin increases endothelial cell growth, migration, and maintains PSCs phenotype [71]. Prokineticin (PK) is another protein secreted by aPSCs, which induces the function of the PK/PKR system in endothelial cells and thereby promotes angiogenesis [71]. Our group has shown earlier that TGF $\beta$-activated hPSCs induced tumor cell growth and endothelial cell tube formation, regulated via the therapeutic microRNAs-199a-3p and microRNA-214-3p [88]. Another study has also shown that PSCs increase endothelial cell tube formation and proliferation via hepatocyte growth factor (HGF)/c-MET/urokinase-type plasminogen activator (UPA) pathway [89].

\subsubsection{Crosstalk with neurons}

PSCs are capable of inducing neuron outgrowth, as was demonstrated by the incubation of dorsal root ganglia with the conditioned medium collected from PSCs derived from human pancreatic cancer [90]. More recently it has been shown that PSCs contribute to pain in pancreatic cancer via sonic hedgehog $(\mathrm{SHH})$ pathway stimulated secretion of neurotrophic factors, such as nerve-growth factor (BGF) and brain-derived neurotrophic factors (BDNF), inducing the secretion of pain factors from dorsal root ganglia [91].

These studies demonstrate that aPSCs aggravate the tumor microenvironment not only by producing ECM but also by establishing a crosstalk with cancer cells and other stromal cells. Disruption into the crosstalk using targeting technologies may provide novel therapeutic options. 
Since conventional therapeutic strategies used for the treatment of PDAC, as chemo- and radiotherapy, only bring minor survival benefits, dampening the tumor supportive function of the tumor stroma by modulating aPSCs seems to be a promising strategy to improve PDAC treatment. On the one hand, therapeutic strategies aiming to deplete PSCs have proven to contribute to the aggressiveness of PDAC rather than contributing to therapeutic benefits. On the other hand, there is convincing evidence that therapeutic strategies that aim at reprogramming of aPSCs hold great promise. The development of aPSC-specific therapeutic strategies should focus on inhibiting the activation of quiescent PSCs and their differentiation into CAFs, which will inhibit the further enhancement of stroma and stroma-induced tumor promoting effects. Another way could be to reverse aPSCs or CAFs into quiescent PSCs, if in any case possible. This would not only impede the aPSC-induced effects immediately, but also start reversing the pro-tumorigenic microenvironment. This will block the effects of secreted growth factor, cytokines and chemokines involved in the crosstalk between PSCs and PDAC cancer cells. Extensive research has been steered in this direction, some of which has been discussed below.

\section{Strategies to modulate aPSCs and CAFs}

352

A number of strategies have been under intense investigation to disrupt or modulate the tumor stroma based on aPSC targeting. These studies are summarized in Table 1.

Sonic Hedgehog pathway: Having the role of the hedgehog pathway in supporting the tumor stroma via paracrine signaling from neoplastic to stromal cells, Olive et al. [92] investigated the effects of a hedgehog inhibitor IPI-926 on the delivery and efficacy of gemcitabine. This combination therapy increased the intra-tumoral vascular density as well as the intra-tumoral concentration of gemcitabine, resulting is a transient stabilization of the disease [92]. Conversely, Rhim et al. [93] demonstrated that deletion of sonic hedgehog in a mouse model of PDAC, resulted in tumors with reduced stroma content. These tumors were more aggressive, exhibited undifferentiated histology, increased vascularity and heightened proliferation compared to controls [93]. Consequently, a follow up phase II clinical trial with hedgehog inhibitor IPI-926 was discontinued due to increased mortality [94]. Similar to IPI926, other stroma-depleting therapeutic strategies did not improve patient survival and in some cases were associated with adverse effects. Özdemir et al. performed a study in which 
a-SMA expressing myofibroblasts were depleted in transgenic mice, resulting in invasive, undifferentiated tumors with enhanced hypoxia, epithelial-to-mesenchymal transition, cancer stem cells and reduced survival [95]. On the one hand, these findings highlight that the stroma has tumor suppressive properties. On the other hand, the negative outcome of stroma depleting studies might be due to the complete removal of fibrotic barriers which hold tumor cells in place and prevents their metastasis/invasiveness. Therefore, modulating the tumor stroma to dampen the tumor promoting activities rather than depleting the stroma could result in therapeutic benefits [96]. More recently, the benefits of this strategy have been demonstrated by us and others $[97,98]$.

PEGylated hyaluronidase: Within PDAC tumor, solid stress has been closely related to drug resistance and therapeutic strategies decreasing solid stress show potential therapeutic benefit [85]. ECM components such as collagen and hyaluronic acid, and aPSCs are the main components of the stroma causing solid stress [14]. A few studies have been performed investigating the effect of the stroma and/or stromal components on drug penetration. Inhibiting hedgehog signaling to deplete tumor stromal tissue could enhance the delivery of chemotherapy in PDAC tumor-bearing mice [92]. Other studies have enzymatically degraded hyaluronic acid in the tumor stroma which resulted in normalized interstitial fluid pressure, re-expansion of the vasculature, increased tumor suppression with gemcitabine and prolonged survival $[45,99]$. The PEGylated hyaluronidase (PEGPH20) has been assessed in combination with gemcitabine, improving survival and attenuating tumor growth in mice when compared with gemcitabine alone [99]. In a phase Ib study, PEGPH20 in combination with gemcitabine showed an increase in progression-free and overall survival rates of patients with metastatic PDAC, but also thromboembolic event in $29 \%$ of patients [100]. PEGPH20 is currently in clinical trials in advanced cancer patients.

MMP inhibitors: Inhibition of MMPs is another interesting therapeutic strategy for the treatment of PDAC. Marimastat, a broad-spectrum MMP inhibitor, was assessed in a randomized clinical trial in which the 1-year survival rate of patients treated with marimastat was similar to those treated with gemcitabine [101]. However, when marimastat was tested as a combination therapy with gemcitabine, it showed no additional benefits compared to gemcitabine [102]. Bay 12-9566, an inhibitor of MMP-3, -9 and -13 , has also been compared 
to gemcitabine in a phase III clinical trial but showed less therapeutic efficacy in advanced PDAC [103].

CTGF inhibitor: Connective tissue growth factor, which is known to induce aPSCs proliferation, migration and ECM production, is another potential therapeutic target [104]. CTGF has been blocked using the monoclonal antibody FG-3019 [105] or antagonist, blocking the interaction between CTGF and chemokine receptors [106]. FG-3019 induced the effectiveness of gemcitabine but did not affect intra-tumoral accumulation of gemcitabine in a mouse model of PDAC [105].

Pirfenidone: Pirfenidone, an anti-fibrotic agent, has been shown to reduce aPSC proliferation, invasion, migration, secretion of collagen and periostin, and decreased overall tumor growth, peritoneal disseminated nodules and liver metastasis in an orthotopic aPSCs and cancer cells co-injection tumor model [107]. When pirfenidone treatment was combined with gemcitabine, tumor growth was significantly attenuated compared to gemcitabine alone [107]. Additionally, pirfenidone has been used in combination with $\mathrm{N}$-acetyl cysteine, and reduced desmoplasia in an orthotopic hamster model, induced with HapT1 pancreatic cancer cells [108].

Angiotensin inhibitors: Two different inhibitors have been used against angiotensin II, which stimulates proliferation of aPSCS through the protein kinase C and EGF-ERK pathway [2]. Olmesartan is an angiotensin II type I receptor blocker, which decreased the proliferation and collagen I synthesis of aPSCs and inhibited the growth and $\alpha$-SMA expression in subcutaneous tumors consisting of AsPc-1 and aPSCs [109]. Another angiotensin II type I receptor inhibitor, losartan, reduced stress in solid tumors, resulting in increased vascular perfusion which enhanced chemotherapy efficiency in pancreatic and breast cancer models [110].

Vitamin A and D analogs: Other strategies focus on reprogramming of aPSCs into their quiescent state to diminish aPSC-induced tumor promotion [2]. When activated, PSCs lose their cytoplasmic vitamin A (retinol) storing lipid droplets. Patients with PDAC are often deficient in vitamin A and D, which supports the activation of PSCs [111] Treatment of aPSC in vitro with all-trans retinoic acid (ATRA) showed inhibitory effects on aPSC migration and collagen synthesis [111]. Additionally, ATRA treatment of aPSCs induced quiescence in PSCs, leading to reduced proliferation and increased apoptosis of surrounding cancer cells [112]. Currently, a phase Ib study is underway investigating ATRA along with gemcitabine and nab- 
paclitaxel in PDAC [113]. More recently, ATRA has been combined with heat shock protein 47 (HSP47) targeting siRNA, capable of reprogramming hPSCs, and delivered using a $\mathrm{pH}$ responsive polyethylene glycol (PEG) grafted polythylamine (PEI)-coated gold nanoparticles [114]. This nanoparticle formulation reprogrammed PSCs and inhibited ECM hyperplasia, causing enhanced drug delivery to orthotopic (hPSC + Panc-1) pancreatic tumors, thereby increasing the efficacy of gemcitabine [114]. Additionally, the vitamin D receptor (VDR) has been shown to be a master transcriptional regulator to regain the quiescent state of PSCs [98]. Calcipotriol, a VDR ligand in combination with gemcitabine, could induce stromal reprogramming in $\mathrm{KRAS}^{\mathrm{G} 12 \mathrm{D} /+}$; $\mathrm{p53}^{\mathrm{R} 172 \mathrm{H} /+}$; PdxCre mice (referred to as KPC mice), increase drug accumulation in tumors, reduce tumor volume and increase survival compared to gemcitabine treatment alone [98].

MicroRNA based approaches: Another interesting class of targets to reprogram aPSCs are microRNAs (miRNAs), small non protein coding single stranded RNA molecules, which regulate posttranscriptional gene expression [115]. A single miRNA sequence can regulate hundreds of target genes and miRNAs can thereby act as oncogenes or tumor suppressors [115]. Therefore, blocking of oncogenic miRNAs with antisense RNA strands shows therapeutic potential [115]. MicroRNA-21 has been observed to be upregulated in CAFs of PDAC and was associated with poor survival [116]. Although the function of miRNA-21 in the stroma has not been understood, it has shown to reduce the growth of MiaPaCa-2 tumors in mice [117]. In TGF- $\beta$-activated PSCs and PDAC biopsies, miRNA-29a and miRNA-29b were found to be decreased [2]. Restoration of miR-29 expression in aPSCs reduced stroma accumulation and tumor growth [73]. We have identified miRNA-199a and miRNA-214 to be overexpressed in CAFs and aPSCs [118]. Additionally, their role in aPSC differentiation, migration, tube formation by endothelial cells, aPSC-induced paracrine effects on tumor cells and growth of 3D-heterospheroids composed of aPSCs and cancer cells has been demonstrated [118].

452 Galectin-1: Recently, genetic deletion of galectin-1 in a KRAS-driven tumor model in mice, resulted in decreased stroma activation, vascularization and increased T-cell infiltration [119]. Activated PSCs-specific depletion of galectin showed an attenuation in metastasis and tumor formation [119]. Therefore, targeting galectin-1 seems to be a promising therapeutic strategy. 
Lipoxin A4: Moreover, we have found that the endogenous lipid lipoxin A4 (LXA4) is capable of inhibiting the activation of human PSCs into CAF-like myofibroblasts in vitro and reduced fibrosis and tumor growth of stroma-rich subcutaneous tumors in vivo [97].

Bromodomain and extraterminal (BET) inhibitors: Bromodomain and extraterminal (BET) family of proteins are shown to be expressed in PSCs within PDAC tumors. BRD4 positively while BRD2 and BRD3 negatively regulates collagen I expression in CAFs [120]. Inhibition using BET inhibitors induce reversion of CAFs phenotype to quiescent phenotype (with reduced fibrosis and collagen I production) thereby inhibited pancreatic tumorigenesis in EL-KrasG ${ }^{12 D}$ transgenic tumor model [120].

\section{Concluding remarks}

The relevance of aPSCs in the progression of PDAC has clearly been demonstrated. Within the tumor microenvironment, activation of PSCs with a vast variety of cytokines and growth factors likely results into different phenotypes of aPSCs or CAFs. Research defining these subtypes will be highly interesting to progress the field further. In particular, identification of tumor-promoting CAFs will help in the quest to design targeted therapies against those cells without affecting tumor suppressive PSCs. This would have the potential to significantly improve the efficacy of existing therapies against PDAC. To develop such strategies to their full potential, it will be of great importance to identify aPSC-derived CAF subpopulations and their significance in PDAC progression and metastasis. Additionally, the identification of markers for tumor-promoting and PSC-derived CAFs will enable the development of therapeutic strategies capable of specifically targeting these cells. Specific markers for tumor promoting CAFs could additionally be used to develop novel diagnostic and prognostic tools.

\section{Acknowledgements}

This work was supported by Swedish research Council project grant (2011-5389).

\section{Competing financial interests:}

J.P. is the founder and stakeholder of ScarTec Therapeutics B.V. 
484 1. Chiaravalli, M. et al. (2017) Pancreatic ductal adenocarcinoma: State-of-the-art 2017 and new 485 therapeutic strategies. Cancer Treat Rev 60, 32-43.

486 2. Kota, J. et al. (2017) Pancreatic cancer: Stroma and its current and emerging targeted therapies. 487 Cancer Lett 391, 38-49.

488 3. Siegel, R.L. et al. (2018) Cancer statistics, 2018. CA Cancer J Clin 68 (1), 7-30.

489 4. Becker, A.E. et al. (2014) Pancreatic ductal adenocarcinoma: risk factors, screening, and early 490 detection. World J Gastroenterol 20 (32), 11182-98.

491 5. Mazur, P.K. and Siveke, J.T. (2012) Genetically engineered mouse models of pancreatic cancer: 492 unravelling tumour biology and progressing translational oncology. Gut 61 (10), 1488-500.

493 6. Apte, M.V. et al. (2013) A starring role for stellate cells in the pancreatic cancer microenvironment. 494 Gastroenterology 144 (6), 1210-9.

495 7. Neesse, A. et al. (2015) Stromal biology and therapy in pancreatic cancer: a changing paradigm. Gut $49664(9), 1476-84$.

497 8. Omary, M.B. et al. (2007) The pancreatic stellate cell: a star on the rise in pancreatic diseases. J Clin 498 Invest $117(1), 50-9$.

499 9. Qian, D. et al. (2017) Galectin-1-driven upregulation of SDF-1 in pancreatic stellate cells promotes 500 pancreatic cancer metastasis. Cancer Lett 397, 43-51.

501 10. Yoshida, N. et al. (2017) Kindlin-2 in pancreatic stellate cells promotes the progression of pancreatic 502 cancer. Cancer Lett 390, 103-114.

503 11. Hessmann, E. et al. (2018) Fibroblast drug scavenging increases intratumoural gemcitabine 504 accumulation in murine pancreas cancer. Gut 67 (3), 497-507.

505 12. Biffi, G. et al. (2018) IL-1-induced JAK/STAT signaling is antagonized by TGF-beta to shape CAF heterogeneity in pancreatic ductal adenocarcinoma. Cancer Discov.

507 13. Allam, A. et al. (2017) Pancreatic stellate cells in pancreatic cancer: In focus. Pancreatology 17 (4), 508 514-522.

509 14. Fu, Y. et al. (2018) The critical roles of activated stellate cells-mediated paracrine signaling, metabolism and onco-immunology in pancreatic ductal adenocarcinoma. Mol Cancer 17 (1), 62.

15. Sinn, M. et al. (2014) alpha-Smooth muscle actin expression and desmoplastic stromal reaction in pancreatic cancer: results from the CONKO-001 study. Br J Cancer 111 (10), 1917-23.

16. Mace, T.A. et al. (2013) Pancreatic cancer-associated stellate cells: A viable target for reducing immunosuppression in the tumor microenvironment. Oncoimmunology 2 (7), e24891.

17. Nagathihalli, N.S. et al. (2016) Pancreatic stellate cell secreted IL-6 stimulates STAT3 dependent invasiveness of pancreatic intraepithelial neoplasia and cancer cells. Oncotarget 7 (40), 65982-65992.

18. Sousa, C.M. et al. (2016) Pancreatic stellate cells support tumour metabolism through autophagic alanine secretion. Nature 536 (7617), 479-83.

19. Phillips, P. (2012) Pancreatic stellate cells and fibrosis. In Pancreatic Cancer and Tumor Microenvironment (Grippo, P.J. and Munshi, H.G. eds).

20. Haqq, J. et al. (2014) Pancreatic stellate cells and pancreas cancer: current perspectives and future strategies. Eur J Cancer 50 (15), 2570-82.

21. Pang, T.C.Y. et al. (2017) Pancreatic stellate cells: what's new? Curr Opin Gastroenterol 33 (5), 366373. 
22. Ota, S. et al. (2016) Involvement of Pancreatic Stellate Cells in Regeneration of Remnant Pancreas after Partial Pancreatectomy. PLoS One 11 (12), e0165747.

23. Ferdek, P.E. et al. (2016) Bile acids induce necrosis in pancreatic stellate cells dependent on calcium entry and sodium-driven bile uptake. J Physiol 594 (21), 6147-6164.

24. Talukdar, R. and Nageshwar Reddy, D. (2013) Is there a single therapeutic target for chronic pancreatitis pain? Gastroenterology 144 (3), e18.

25. Akanuma, N. et al. (2017) Paracrine Secretion of Transforming Growth Factor beta by Ductal Cells Promotes Acinar-to-Ductal Metaplasia in Cultured Human Exocrine Pancreas Tissues. Pancreas 46 (9), 1202-1207.

26. Xue, J. et al. (2015) Alternatively activated macrophages promote pancreatic fibrosis in chronic pancreatitis. Nat Commun 6, 7158.

27. Bynigeri, R.R. et al. (2017) Pancreatic stellate cell: Pandora's box for pancreatic disease biology. World J Gastroenterol 23 (3), 382-405.

28. Binkley, C.E. et al. (2004) The molecular basis of pancreatic fibrosis: common stromal gene expression in chronic pancreatitis and pancreatic adenocarcinoma. Pancreas 29 (4), 254-63.

29. Ling, S. et al. (2014) Inflammation to cancer: The molecular biology in the pancreas (Review). Oncol Lett 7 (6), 1747-1754.

30. Ohlund, D. et al. (2017) Distinct populations of inflammatory fibroblasts and myofibroblasts in pancreatic cancer. J Exp Med 214 (3), 579-596.

31. Ikenaga, N. et al. (2010) CD10+ pancreatic stellate cells enhance the progression of pancreatic cancer. Gastroenterology 139 (3), 1041-51, 1051 e1-8.

32. Franco-Barraza, J. et al. (2017) Matrix-regulated integrin alphavbeta5 maintains alpha5beta1dependent desmoplastic traits prognostic of neoplastic recurrence. Elife 6 .

33. Kuninty, P.R. et al. (2018) Targeting integrin alpha5 receptor in pancreatic stellate cells to diminish tumor-promoting effects in pancreatic cancer. Biorxiv, doi.org/10.1101/350678.

34. Basturk, O. et al. (2015) A Revised Classification System and Recommendations From the Baltimore Consensus Meeting for Neoplastic Precursor Lesions in the Pancreas. Am J Surg Pathol 39 (12), 173041.

35. Oldfield, L.E. et al. (2017) Molecular Events in the Natural History of Pancreatic Cancer. Trends Cancer 3 (5), 336-346.

36. Distler, M. et al. (2014) Precursor lesions for sporadic pancreatic cancer: PanIN, IPMN, and MCN. Biomed Res Int 2014, 474905.

37. Bae, Y.H. et al. (2013) Cancer targeted drug delivery : an elusive dream, Springer.

38. McCarroll, J.A. et al. (2014) Role of pancreatic stellate cells in chemoresistance in pancreatic cancer. Front Physiol 5, 141.

39. Amrutkar, M. and Gladhaug, I.P. (2017) Pancreatic Cancer Chemoresistance to Gemcitabine. Cancers (Basel) 9 (11).

40. Xu, Z. et al. (2010) Role of pancreatic stellate cells in pancreatic cancer metastasis. Am J Pathol 177 (5), 2585-96.

41. Endo, S. et al. (2017) Autophagy inhibition enhances antiproliferative effect of salinomycin in pancreatic cancer cells. Pancreatology 17 (6), 990-996.

42. Berchtold, S. et al. (2015) Collagen type $V$ promotes the malignant phenotype of pancreatic ductal adenocarcinoma. Cancer Lett 356 (2 Pt B), 721-32. 
43. Di Maggio, F. et al. (2016) Pancreatic stellate cells regulate blood vessel density in the stroma of pancreatic ductal adenocarcinoma. Pancreatology 16 (6), 995-1004.

44. Hesler, R.A. et al. (2016) TGF-beta-induced stromal CYR61 promotes resistance to gemcitabine in pancreatic ductal adenocarcinoma through downregulation of the nucleoside transporters hENT1 and hCNT3. Carcinogenesis 37 (11), 1041-1051.

45. Provenzano, P.P. et al. (2012) Enzymatic targeting of the stroma ablates physical barriers to treatment of pancreatic ductal adenocarcinoma. Cancer Cell 21 (3), 418-29.

46. Kamphorst, J.J. et al. (2015) Human pancreatic cancer tumors are nutrient poor and tumor cells actively scavenge extracellular protein. Cancer Res 75 (3), 544-53.

47. Perera, R.M. and Bardeesy, N. (2015) Pancreatic Cancer Metabolism: Breaking It Down to Build It Back Up. Cancer Discov 5 (12), 1247-61.

48. An, M.X. et al. (2017) BAG3 directly stabilizes Hexokinase 2 mRNA and promotes aerobic glycolysis in pancreatic cancer cells. J Cell Biol 216 (12), 4091-4105.

49. Fu, Y. et al. (2017) The reverse Warburg effect is likely to be an Achilles' heel of cancer that can be exploited for cancer therapy. Oncotarget 8 (34), 57813-57825.

50. Wu, Y.S. et al. (2016) Soluble factors from stellate cells induce pancreatic cancer cell proliferation via Nrf2-activated metabolic reprogramming and ROS detoxification. Oncotarget 7 (24), 36719-36732.

51. Halbrook, C.J. and Lyssiotis, C.A. (2017) Employing Metabolism to Improve the Diagnosis and Treatment of Pancreatic Cancer. Cancer Cell 31 (1), 5-19.

52. Tape, C.J. et al. (2016) Oncogenic KRAS Regulates Tumor Cell Signaling via Stromal Reciprocation. Cell 165 (4), 910-20.

53. Commisso, C. et al. (2013) Macropinocytosis of protein is an amino acid supply route in Rastransformed cells. Nature 497 (7451), 633-7.

54. Zhao, H. et al. (2016) Tumor microenvironment derived exosomes pleiotropically modulate cancer cell metabolism. Elife 5, e10250.

55. Schnittert, J. et al. (2017) Anti-microRNA targeting using peptide-based nanocomplexes to inhibit differentiation of human pancreatic stellate cells. Nanomedicine (Lond).

56. Lugea, A. and Waldron, R.T. (2017) Exosome-Mediated Intercellular Communication Between Stellate Cells and Cancer Cells in Pancreatic Ductal Adenocarcinoma. Pancreas 46 (1), 1-4.

57. Saha, S. et al. (2016) Gold Nanoparticle Reprograms Pancreatic Tumor Microenvironment and Inhibits Tumor Growth. ACS Nano 10 (12), 10636-10651.

58. Cao, F. et al. (2015) HES 1 is essential for chemoresistance induced by stellate cells and is associated with poor prognosis in pancreatic cancer. Oncol Rep 33 (4), 1883-9.

59. Ferdek, P.E. and Jakubowska, M.A. (2017) Biology of pancreatic stellate cells-more than just pancreatic cancer. Pflugers Arch 469 (9), 1039-1050.

60. Liu, Y. et al. (2016) Periostin promotes the chemotherapy resistance to gemcitabine in pancreatic cancer. Tumour Biol 37 (11), 15283-15291.

61. Schnittert, J. et al. (2018) Integrins in wound healing, fibrosis and tumor stroma: High potential targets for therapeutics and drug delivery. Adv Drug Deliv Rev 129, 37-53.

62. Hwang, R.F. et al. (2008) Cancer-associated stromal fibroblasts promote pancreatic tumor progression. Cancer Res 68 (3), 918-26.

63. Strell, C. et al. (2017) Stroma-regulated HMGA2 is an independent prognostic marker in PDAC and AAC. Br J Cancer 117 (1), 65-77. 
64. Vonlaufen, A. et al. (2008) Pancreatic stellate cells: partners in crime with pancreatic cancer cells. Cancer Res 68 (7), 2085-93.

65. Vonlaufen, A. et al. (2008) Pancreatic stellate cells and pancreatic cancer cells: an unholy alliance. Cancer Res 68 (19), 7707-10.

66. Levental, K.R. et al. (2009) Matrix crosslinking forces tumor progression by enhancing integrin signaling. Cell 139 (5), 891-906.

67. Robinson, B.K. et al. (2016) Quantitative analysis of 3D extracellular matrix remodelling by pancreatic stellate cells. Biol Open 5 (6), 875-82.

68. Drifka, C.R. et al. (2016) Human pancreatic stellate cells modulate 3D collagen alignment to promote the migration of pancreatic ductal adenocarcinoma cells. Biomed Microdevices 18 (6), 105.

69. Sarper, M. et al. (2016) ATRA modulates mechanical activation of TGF-beta by pancreatic stellate cells. Sci Rep 6, 27639.

70. Chronopoulos, A. et al. (2016) ATRA mechanically reprograms pancreatic stellate cells to suppress matrix remodelling and inhibit cancer cell invasion. Nat Commun 7, 12630.

71. Tang, D. et al. (2013) Persistent activation of pancreatic stellate cells creates a microenvironment favorable for the malignant behavior of pancreatic ductal adenocarcinoma. Int J Cancer 132 (5), 9931003.

72. Schnittert, J. et al. (2019) Integrin alpha11 in pancreatic stellate cells regulates tumor stroma interaction in pancreatic cancer. FASEB J accepted.

73. Kikuta, K. et al. (2010) Pancreatic stellate cells promote epithelial-mesenchymal transition in pancreatic cancer cells. Biochem Biophys Res Commun 403 (3-4), 380-4.

74. Valle, S. et al. (2018) The Ever-Evolving Concept of the Cancer Stem Cell in Pancreatic Cancer. Cancers (Basel) 10 (2).

75. Dorado, J. et al. (2011) Pancreatic cancer stem cells: new insights and perspectives. J Gastroenterol $46(8), 966-73$.

76. Hermann, P.C. et al. (2009) Pancreatic cancer stem cells--insights and perspectives. Expert Opin Biol Ther 9 (10), 1271-8.

77. Rao, C.V. and Mohammed, A. (2015) New insights into pancreatic cancer stem cells. World J Stem Cells 7 (3), 547-55.

78. Magee, J.A. et al. (2012) Cancer stem cells: impact, heterogeneity, and uncertainty. Cancer Cell 21 (3), 283-96.

79. Hermann, P.C. et al. (2007) Distinct populations of cancer stem cells determine tumor growth and metastatic activity in human pancreatic cancer. Cell Stem Cell 1 (3), 313-23.

80. Hamada, S. et al. (2012) Pancreatic stellate cells enhance stem cell-like phenotypes in pancreatic cancer cells. Biochem Biophys Res Commun 421 (2), 349-54.

81. $\mathrm{Wu}, \mathrm{Q}$. et al. (2017) Functions of pancreatic stellate cell-derived soluble factors in the microenvironment of pancreatic ductal carcinoma. Oncotarget 8 (60), 102721-102738.

82. Gabitass, R.F. et al. (2011) Elevated myeloid-derived suppressor cells in pancreatic, esophageal and gastric cancer are an independent prognostic factor and are associated with significant elevation of the Th2 cytokine interleukin-13. Cancer Immunol Immunother 60 (10), 1419-30.

83. Markowitz, J. et al. (2015) Patients with pancreatic adenocarcinoma exhibit elevated levels of myeloid-derived suppressor cells upon progression of disease. Cancer Immunol Immunother 64 (2), 149-59. 
84. Mace, T.A. et al. (2013) Pancreatic cancer-associated stellate cells promote differentiation of myeloid-derived suppressor cells in a STAT3-dependent manner. Cancer Res 73 (10), 3007-18.

85. Incio, J. et al. (2016) Obesity-Induced Inflammation and Desmoplasia Promote Pancreatic Cancer Progression and Resistance to Chemotherapy. Cancer Discov 6 (8), 852-69.

86. Tang, D. et al. (2012) High expression of Galectin-1 in pancreatic stellate cells plays a role in the development and maintenance of an immunosuppressive microenvironment in pancreatic cancer. Int J Cancer 130 (10), 2337-48.

87. Ene-Obong, A. et al. (2013) Activated pancreatic stellate cells sequester CD8+ T cells to reduce their infiltration of the juxtatumoral compartment of pancreatic ductal adenocarcinoma. Gastroenterology 145 (5), 1121-32.

88. Kuninty, P.R. et al. (2016) MicroRNA-199a and-214 as potential therapeutic targets in pancreatic stellate cells in pancreatic tumor. Oncotarget 7 (13), 16396-16408.

89. Patel, M.B. et al. (2014) The role of the hepatocyte growth factor/c-MET pathway in pancreatic stellate cell-endothelial cell interactions: antiangiogenic implications in pancreatic cancer. Carcinogenesis 35 (8), 1891-900.

90. Demir, I.E. et al. (2010) The microenvironment in chronic pancreatitis and pancreatic cancer induces neuronal plasticity. Neurogastroenterol Motil 22 (4), 480-90, e112-3.

91. Han, L. et al. (2016) Pancreatic stellate cells contribute pancreatic cancer pain via activation of sHH signaling pathway. Oncotarget 7 (14), 18146-58.

92. Olive, K.P. et al. (2009) Inhibition of Hedgehog signaling enhances delivery of chemotherapy in a mouse model of pancreatic cancer. Science 324 (5933), 1457-61.

93. Rhim, A.D. et al. (2014) Stromal elements act to restrain, rather than support, pancreatic ductal adenocarcinoma. Cancer Cell 25 (6), 735-47.

94. Amakye, D. et al. (2013) Unraveling the therapeutic potential of the Hedgehog pathway in cancer. Nat Med 19 (11), 1410-22.

95. Ozdemir, B.C. et al. (2015) Depletion of Carcinoma-Associated Fibroblasts and Fibrosis Induces Immunosuppression and Accelerates Pancreas Cancer with Reduced Survival. Cancer Cell 28 (6), 831833.

96. Prakash, J. (2016) Cancer-Associated Fibroblasts: Perspectives in Cancer Therapy. Trends Cancer 2 (6), 277-279.

97. Schnittert, J. et al. (2018) Reprogramming tumor stroma using an endogenous lipid lipoxin A4 to treat pancreatic cancer. Cancer Lett 420, 247-258.

98. Sherman, M.H. et al. (2014) Vitamin D Receptor-Mediated Stromal Reprogramming Suppresses Pancreatitis and Enhances Pancreatic Cancer Therapy. Cell 159 (1), 80-93.

99. Jacobetz, M.A. et al. (2013) Hyaluronan impairs vascular function and drug delivery in a mouse model of pancreatic cancer. Gut 62 (1), 112-20.

100. Hingorani, S.R. et al. (2016) Phase Ib Study of PEGylated Recombinant Human Hyaluronidase and Gemcitabine in Patients with Advanced Pancreatic Cancer. Clin Cancer Res 22 (12), 2848-54.

101. Bramhall, S.R. et al. (2001) Marimastat as first-line therapy for patients with unresectable pancreatic cancer: a randomized trial. J Clin Oncol 19 (15), 3447-55.

102. Bramhall, S.R. et al. (2002) A double-blind placebo-controlled, randomised study comparing gemcitabine and marimastat with gemcitabine and placebo as first line therapy in patients with advanced pancreatic cancer. Br J Cancer 87 (2), 161-7. 
103. Moore, M.J. et al. (2003) Comparison of gemcitabine versus the matrix metalloproteinase inhibitor BAY 12-9566 in patients with advanced or metastatic adenocarcinoma of the pancreas: a phase III trial of the National Cancer Institute of Canada Clinical Trials Group. J Clin Oncol 21 (17), 3296302.

104. Charrier, A. and Brigstock, D.R. (2013) Regulation of pancreatic function by connective tissue growth factor (CTGF, CCN2). Cytokine Growth Factor Rev 24 (1), 59-68.

105. Neesse, A. et al. (2013) CTGF antagonism with mAb FG-3019 enhances chemotherapy response without increasing drug delivery in murine ductal pancreas cancer. Proc Natl Acad Sci U S A 110 (30), 12325-30.

106. Ijichi, H. et al. (2011) Inhibiting Cxcr2 disrupts tumor-stromal interactions and improves survival in a mouse model of pancreatic ductal adenocarcinoma. J Clin Invest 121 (10), 4106-17.

107. Kozono, S. et al. (2013) Pirfenidone inhibits pancreatic cancer desmoplasia by regulating stellate cells. Cancer Res 73 (7), 2345-56.

108. Suklabaidya, S. et al. (2016) Characterization and use of HapT1-derived homologous tumors as a preclinical model to evaluate therapeutic efficacy of drugs against pancreatic tumor desmoplasia. Oncotarget 7 (27), 41825-41842.

109. Masamune, A. et al. (2013) The angiotensin II type I receptor blocker olmesartan inhibits the growth of pancreatic cancer by targeting stellate cell activities in mice. Scand J Gastroenterol 48 (5), 602-9.

110. Chauhan, V.P. et al. (2013) Angiotensin inhibition enhances drug delivery and potentiates chemotherapy by decompressing tumour blood vessels. Nat Commun 4, 2516.

111. Jaster, R. et al. (2003) Regulation of pancreatic stellate cell function in vitro: biological and molecular effects of all-trans retinoic acid. Biochem Pharmacol 66 (4), 633-41.

112. Froeling, F.E. et al. (2011) Retinoic acid-induced pancreatic stellate cell quiescence reduces paracrine Wnt-beta-catenin signaling to slow tumor progression. Gastroenterology 141 (4), 1486-97, 1497 e1-14.

113. Kocher, $\mathrm{H}$. et al. STAR_PAC: A Phase $1 \mathrm{~B}$ study repurposing ATRA as stromal targeting agent along with gemcitabine and nab\&\#xac;Paclitaxel for pancreatic cancer. Pancreatology 16 (3), S4-S5.

114. Han, X. et al. (2018) Reversal of pancreatic desmoplasia by re-educating stellate cells with a tumour microenvironment-activated nanosystem. Nat Commun 9 (1), 3390.

115. Kuninty, P.R. et al. (2016) MicroRNA Targeting to Modulate Tumor Microenvironment. Front Oncol 6, 3.

116. Kadera, B.E. et al. (2013) MicroRNA-21 in pancreatic ductal adenocarcinoma tumor-associated fibroblasts promotes metastasis. PLoS One 8 (8), e71978.

117. Sicard, F. et al. (2013) Targeting miR-21 for the therapy of pancreatic cancer. Mol Ther 21 (5), 98694.

118. Kuninty, P.R. et al. (2016) MicroRNA-199a and -214 as potential therapeutic targets in pancreatic stellate cells in pancreatic tumor. Oncotarget 7 (13), 16396-408.

119. Orozco, C.A. et al. (2018) Targeting galectin-1 inhibits pancreatic cancer progression by modulating tumor-stroma crosstalk. Proc Natl Acad Sci U S A 115 (16), E3769-E3778.

120. Kumar, K. et al. (2017) BET inhibitors block pancreatic stellate cell collagen I production and attenuate fibrosis in vivo. JCl Insight 2 (3), e88032. 
739 121. Banerjee, S. et al. (2016) Impaired Synthesis of Stromal Components in Response to Minnelide 740 Improves Vascular Function, Drug Delivery, and Survival in Pancreatic Cancer. Clin Cancer Res 22 (2), $741 \quad 415-25$.

742

743

744

745 


\begin{tabular}{|c|c|c|c|c|}
\hline $\begin{array}{l}\text { Therapeutic } \\
\text { Strategy }\end{array}$ & $\begin{array}{l}\text { Co- } \\
\text { treatment }\end{array}$ & $\begin{array}{l}\text { Stage } \\
\text { (year) }\end{array}$ & Function & Ref. \\
\hline \multicolumn{5}{|c|}{ Clinical Studies } \\
\hline $\begin{array}{l}\mathrm{PH} 20 \\
\text { hyaluronidase }\end{array}$ & & Phase-lb & $\begin{array}{l}\text { Increased progression-free and overall survival } \\
\text { rates of patients with metastatic PDAC, but also } \\
\text { thromboembolic event in } 29 \% \text { of patients. }\end{array}$ & [100] \\
\hline Drug: IPI-926 & $\begin{array}{l}\text { Gemcitabi } \\
\text { ne }\end{array}$ & $\begin{array}{l}\text { Phase II } \\
\text { (2012) }\end{array}$ & Discontinued due to increased mortality & [94] \\
\hline Marimastat & / & $\begin{array}{l}\text { Phase II } \\
\text { (2001) }\end{array}$ & $\begin{array}{l}\text { 1-year survival rate of patients treated with } \\
\text { marimastat was similar to those treated with } \\
\text { gemcitabine. }\end{array}$ & {$[101]$} \\
\hline Marimastat & $\begin{array}{l}\text { Gemcitabi } \\
\text { ne }\end{array}$ & $\begin{array}{l}\text { Phase II } \\
\text { (2001) }\end{array}$ & $\begin{array}{l}\text { No additional benefits compared to gemcitabine } \\
\text { alone. }\end{array}$ & [102] \\
\hline Bay 12-9566 & / & $\begin{array}{l}\text { Phase III } \\
\text { (2003) }\end{array}$ & $\begin{array}{l}\text { Inhibitor of MMP-3, }-9 \text { and }-13 \text {, showed less } \\
\text { therapeutic efficacy in advanced PDAC compared } \\
\text { to gemcitabine. }\end{array}$ & [103] \\
\hline ATRA & $\begin{array}{l}\text { Gemcitabi } \\
\text { ne and } \\
\text { nab- } \\
\text { paclitaxel }\end{array}$ & Phase IB & $\begin{array}{l}\text { Investigating ATRA along with gemcitabine and } \\
\text { nab-paclitaxel in PDAC. }\end{array}$ & [113] \\
\hline \multicolumn{5}{|c|}{ Pre-clinical } \\
\hline Drug: IPI-926 & $\begin{array}{l}\text { Gemcitabi } \\
\text { ne }\end{array}$ & $\begin{array}{l}\text { Pre- } \\
\text { clinical } \\
\text { (2009) }\end{array}$ & $\begin{array}{l}\text { Hedgehog inhibitor. Increased intra-tumoral } \\
\text { vascular density and the intra-tumoral } \\
\text { concentration of gemcitabine, resulting is a } \\
\text { transient disease stabilization. }\end{array}$ & {$[92]$} \\
\hline Minnelide & / & $\begin{array}{l}\text { Pre- } \\
\text { clinical } \\
\text { (2015) }\end{array}$ & $\begin{array}{l}\text { HSP70 inhibitor. Reduced ECM components like HA } \\
\text { and collagen, improved functional vasculature, } \\
\text { increased intra-tumoral drug delivery and } \\
\text { decreased viability of tumor cells and stromal cells } \\
\text { in experimental models of pancreatic tumor. }\end{array}$ & {$[121]$} \\
\hline
\end{tabular}




\begin{tabular}{|c|c|c|c|c|}
\hline $\begin{array}{l}\text { BET inhibitors (JQ1 } \\
\text { and I-BET151) }\end{array}$ & / & $\begin{array}{l}\text { Pre- } \\
\text { clinical } \\
(2017)\end{array}$ & $\begin{array}{l}\text { BET inhibitors induced PSCs quiescence, } \\
\text { attenuated fibrosis and collagen I production in the } \\
\text { EL-Kras }^{\mathrm{G} 2 \mathrm{D}} \text { transgenic mouse model. }\end{array}$ & [120] \\
\hline $\begin{array}{l}\text { Genetic depletion } \\
\text { of } \quad \alpha \text {-SMA } \\
\text { expressing cells }\end{array}$ & / & $\begin{array}{l}\text { Pre- } \\
\text { clinical } \\
(2015)\end{array}$ & $\begin{array}{l}\text { Invasive, undifferentiated tumors with enhanced } \\
\text { hypoxia, epithelial-to-mesenchymal transition, } \\
\text { cancer stem cells and reduced survival. }\end{array}$ & [95] \\
\hline $\begin{array}{l}\text { Genetic deletion of } \\
\text { Shh }\end{array}$ & / & $\begin{array}{l}\text { Pre- } \\
\text { clinical } \\
(2014)\end{array}$ & $\begin{array}{l}\text { More aggressive tumors with, undifferentiated } \\
\text { histology, increased vascularity and heightened } \\
\text { proliferation. }\end{array}$ & [93] \\
\hline IL-1 $\beta$ inhibition & / & $\begin{array}{l}\text { Pre- } \\
\text { clinical } \\
(2016)\end{array}$ & Reduced obesity related tumor growth & [85] \\
\hline $\begin{array}{l}\mathrm{PH} 20 \\
\text { hyaluronidase }\end{array}$ & $\begin{array}{l}\text { Gemcitabi } \\
\text { ne }\end{array}$ & $\begin{array}{l}\text { Pre- } \\
\text { clinical } \\
(2012)\end{array}$ & $\begin{array}{l}\text { Triggered fenestrations and } \\
\text { Inter-endothelial junctional gaps in PDAC tumor } \\
\text { endothelia, promoted a tumor-specific increase in } \\
\text { macromolecular permeability, inhibited tumor } \\
\text { growth and prolonged survival. }\end{array}$ & [99] \\
\hline $\begin{array}{l}\text { PH2O } \\
\text { hyaluronidase }\end{array}$ & $\begin{array}{l}\text { Gemcitabi } \\
\text { ne }\end{array}$ & $\begin{array}{l}\text { Pre- } \\
\text { clinical } \\
(2012)\end{array}$ & $\begin{array}{l}\text { Ablated stromal hyaluronic acid, normalization of } \\
\text { interstitial fluid pressure, re-expansion of } \\
\text { vasculature, permanent remodeling of the tumor } \\
\text { microenvironment and prolonged survival. }\end{array}$ & [45] \\
\hline $\begin{array}{l}\text { Monoclonal } \\
\text { antibody FG-3019 }\end{array}$ & $\begin{array}{l}\text { Gemcitabi } \\
\text { ne }\end{array}$ & $\begin{array}{l}\text { Pre- } \\
\text { clinical } \\
(2013)\end{array}$ & $\begin{array}{l}\text { Induced the effectiveness of gemcitabine while not } \\
\text { affecting intra-tumoral accumulation of } \\
\text { gemcitabine in a KPC mouse model of PDAC. }\end{array}$ & [105] \\
\hline Pirfenidone & $\begin{array}{l}\text { Gemcitabi } \\
\text { ne }\end{array}$ & $\begin{array}{l}\text { Pre- } \\
\text { clinical } \\
(2013)\end{array}$ & $\begin{array}{l}\text { Reduction of aPSC proliferation, invasion, } \\
\text { migration, secretion of collagen and periostin and } \\
\text { decreased overall tumor growth, peritoneal } \\
\text { disseminated nodules and liver metastasis in an } \\
\text { othotopic mouse model induced with aPSCs and } \\
\text { cancer cells. Attenuated tumor growth compared } \\
\text { to gemcitabine alone. }\end{array}$ & [107] \\
\hline
\end{tabular}




\begin{tabular}{|c|c|c|c|c|}
\hline Pirfenidone & $\begin{array}{l}\mathrm{N} \text {-acetyl } \\
\text { cysteine }\end{array}$ & $\begin{array}{l}\text { Pre- } \\
\text { clinical } \\
(2016)\end{array}$ & $\begin{array}{l}\text { Reduced desmoplasia in an orthotopic hamster } \\
\text { model, induced with HapT1 pancreatic cancer cells. }\end{array}$ & [108] \\
\hline Olmesartan & / & $\begin{array}{l}\text { Pre- } \\
\text { clinical } \\
(2013)\end{array}$ & $\begin{array}{l}\text { Decreased the proliferation and collagen I } \\
\text { synthesis of aPSCs and inhibited the growth and } \alpha \text { - } \\
\text { SMA expression in subcutaneous tumors consisting } \\
\text { of AsPc-1 and aPSCs. }\end{array}$ & [109] \\
\hline Losartan & / & $\begin{array}{l}\text { Pre- } \\
\text { clinical } \\
(2013)\end{array}$ & $\begin{array}{l}\text { Reduced stress in solid tumors, resulting in } \\
\text { increased vascular perfusion which enhanced } \\
\text { chemotherapy efficiency. }\end{array}$ & [110] \\
\hline $\begin{array}{l}\text { All-trans retinoic } \\
\text { acid (ATRA) }\end{array}$ & / & $\begin{array}{l}\text { Pre- } \\
\text { clinical } \\
(2003 \\
2011)\end{array}$ & $\begin{array}{l}\text { Showed inhibitory effects on aPSC migration and } \\
\text { collagen synthesis and induced quiescence in PSCs, } \\
\text { leading to a reduction in their proliferation and } \\
\text { increased apoptosis of surrounding cancer cells. }\end{array}$ & $\begin{array}{l}{[111,} \\
112]\end{array}$ \\
\hline $\begin{array}{l}\text { Nanoparticles (All- } \\
\text { trans retinoic acid } \\
\text { (ATRA) + siRNA } \\
\text { HSP47) }\end{array}$ & $\begin{array}{l}\text { Gemcitabi } \\
\text { ne }\end{array}$ & $\begin{array}{l}\text { Pre-clinical } \\
(2018)\end{array}$ & $\begin{array}{l}\text { Reprogrammed PSCs and inhibited ECM hyperplasia, } \\
\text { causing enhanced drug delivery to orthotopic (hPSC + } \\
\text { Panc-1) pancreatic tumors, resulting in increased } \\
\text { efficacy of gemcitabine }\end{array}$ & [114] \\
\hline Calcipotriol & $\begin{array}{l}\text { Gemcitabi } \\
\text { ne }\end{array}$ & $\begin{array}{l}\text { Pre- } \\
\text { clinical } \\
(2014)\end{array}$ & $\begin{array}{l}\text { Induces stromal reprogramming in KPC mice, } \\
\text { increase drug accumulation in tumors, reduce } \\
\text { tumor volume and increase survival compared to } \\
\text { gemcitabine treatment alone. }\end{array}$ & [98]. \\
\hline MicroRNA-29 & / & $\begin{array}{l}\text { Pre- } \\
\text { clinical } \\
(2010)\end{array}$ & $\begin{array}{l}\text { Restoration of miR-29 expression in aPSCs reduced } \\
\text { stroma accumulation and tumor growth. }\end{array}$ & [73] \\
\hline $\begin{array}{l}\text { MicroRNA-199a, } \\
\text { microRNA-214 }\end{array}$ & / & $\begin{array}{l}\text { Pre- } \\
\text { clinical } \\
(2016)\end{array}$ & $\begin{array}{l}\text { Inhibits aPSC differentiation, migration, tube } \\
\text { formation by endothelial cells, aPSC-induced } \\
\text { paracrine effects on tumor cells and growth of 3D- } \\
\text { heterospheroids composed of aPSCs and cancer } \\
\text { cells has been demonstrated }\end{array}$ & [118] \\
\hline
\end{tabular}




\begin{tabular}{|l|l|l|l|l|}
\hline Lipoxin A4 (LXA4) & $/$ & $\begin{array}{l}\text { Pre- } \\
\text { clinical } \\
(2018)\end{array}$ & $\begin{array}{l}\text { Attenuates the activation of human PSCs into CAF- } \\
\text { like myofibroblasts in vitro and reduces fibrosis and } \\
\text { tumor growth of stroma-rich subcutaneous tumors } \\
\text { in vivo. }\end{array}$ & [97] \\
\hline
\end{tabular}

